\title{
Evaluation of the effect of mitral stenosis severity on the left ventricular systolic function using isovolumic myocardial acceleration
}

\author{
Mehmet Erturk, Hale Unal Aksu, Omer Celik, Fatih Uzun, Ozgur Akgul, \\ Hamdi Pusuroglu, Ali Kemal Kalkan, Ahmet Arif Yalcin, \\ Muhammet Hulusi Satilmisoglu, Mustafa Kemal Erol \\ Department of Cardiology, Istanbul Mehmet Akif Ersoy Thoracic and Cardiovascular \\ Surgery Training and Research Hospital, Istanbul, Turkey
}

\begin{abstract}
Background: Isovolumic acceleration (IVA) is a new tissue Doppler parameter in the assessment of systolic function of both left and right ventricles. It remains unaffected with the changes in pre-and after-load within the physiological range. The aim of our study was to assess the effect of mitral stenosis degree, which is determined by echocardiography, on the left ventricular (LV) function using IVA.

Methods: A total number of 62 patients with mitral stenosis (MS) and 32 healthy controls were examined. The severity of MS (mild, moderate, and severe) was determined on the basis of mitral valve area (MVA) and the mean diastolic mitral gradient findings. The peak myocardial velocities during isovolumic contraction, systole, early diastole and late diastole were measured by using tissue Doppler imaging (TDI).

Results: All TDI-derived global LV basal wall systolic (peak myocardial isovolumic contraction velocity, peak myocardial systolic velocity and IVA), and diastolic velocities (peak early and late diastolic velocities) were significantly decreased in the patients with MS, compared to the healthy patients ( $p<0.001$, for all). However, IVA was not different when the degree of $M S$ was evaluated $(p=0.114)$. In addition, IVA was not correlated with the MVA $(r=0.185$, $p=0.150$ ).
\end{abstract}

Conclusions: Left ventricular function is impaired in patients with MS regardless of the severity of the disease. (Cardiol J 2014; 21, 4: 442-448)

Key words: isovolumic acceleration, mitral stenosis, left ventricular function, tissue Doppler imaging

\section{Introduction}

Mitral stenosis (MS) still leads to significant morbidity and mortality worldwide [1]. The presence of impaired left ventricular (LV) systolic function, determined by M-mode and/or two- -dimensional (2D) echocardiography (ECHO), is noted in $25-30 \%$ of the patients with MS [2-6]. Recently, in the studies using tissue Doppler imaging (TDI) [7-9] and strain/strain rate imaging [10, 11], which are more sensitive methods compared to the conventional ECHO, the occurrence of subclinical

Address for correspondence: Mehmet Erturk, MD, Kardiyoloji Kliniği, Mehmet Akif Ersoy Göğüs Kalp ve Damar Cerrahisi, Eğitim ve Araştırma Hastanesi, İstasyon Mah. Turgut Özal Bulvarı No: 11 Küçükçekmece, 34303, Istanbul, Turkey, tel: +90212 69220 00, fax: +9021247194 94, e-mail: drerturk@gmail.com

Received: 19.06.2013 Accepted: 25.06.2013 
LV systolic dysfunction was confirmed even in MS patients with preserved ejection fraction $(\mathrm{EF})$. The functional and/or myocardial factors, which cause impaired LV systolic function, are unclear. In the study of Lee and Lee [12], systolic dysfunction of LV was suggested to be associated with the severity of myocardial involvement in isolated mitral valve stenosis during the rheumatic attack, not with the degree of the MS demonstrated by ECHO. Furthermore, Sengupta et al. [8] showed that subclinical LV dysfunction improved following the percutaneous mitral valvuloplasty.

Isovolumic acceleration (IVA) is a new tissue Doppler parameter for the assessment of systolic function of both left and the right ventricles $[13,14]$. IVA is calculated as a ratio of tissue Doppler-derived peak myocardial velocity during isovolumetric contraction (IVV) divided by the acceleration time (AT). This parameter has been validated in a variety of experimental $[13,14]$ and clinical $[15,16]$ settings. IVA remains unaffected by the changes in the preload and afterload within the physiological range [13-16]. It can detect small changes in the contractile function and is well correlated with the invasive or noninvasive measures of $\mathrm{LV} \mathrm{dP/dt}[13,17]$.

Effect of the degree of mitral valve stenosis, which is determined by echocardiographic and hemodynamic parameters, on LV function is not clearly evaluated. The aim of our study was to assess the effect of mitral valve stenosis degree, which is determined by ECHO, on the LV function using IVA.

\section{Methods}

A total number of 94 subjects, 62 isolated mitral valve stenosis patients with preserved LVEF and sinus rhythm (mean age $39 \pm 8$ years; 56 [90.3\%] females) and 32 healthy volunteers (mean age $36 \pm 8$ years; 56 [81.2\%] females) were included in our study. All the participants underwent both conventional echocardiography and TDI. The patients with MS were divided into three groups (mild, moderate and severe), based on their mitral valve area (MVA) determined by ECHO, and mean diastolic mitral gradients: 21 patients with mild stenosis (MVA $>1.5 \mathrm{~cm}^{2}$, mean gradient $<5 \mathrm{~mm} \mathrm{Hg}$ ), 21 patients with moderate stenosis (MVA $=1-$ $-1.5 \mathrm{~cm}^{2}$, mean gradient $\left.=5-10 \mathrm{~mm} \mathrm{Hg}\right)$ and 20 patients with severe stenosis (MVA $<1 \mathrm{~cm}^{2}$, mean gradient $>10 \mathrm{~mm} \mathrm{Hg}$ ).

Patients who had coronary artery disease, moderate to severe aortic and mitral regurgitation, aortic stenosis, hyperthyroidism, chronic obstructi- ve pulmonary disease, atrioventricular conduction abnormality, left bundle branch block, segmental wall motion abnormalities, and severely calcified mitral valve structure were excluded in this study.

Written informed consent form was obtained from the patients following approval of the study by the institutional review board. The study was consistent with the Declaration of Helsinki.

\section{Conventional echocardiographic examination}

All the transthoracic echocardiographic (TTE) examinations were performed using GE vivid S6 Vingmed system 5 (Norway, Horten) equipped with $2.5-4 \mathrm{MHz}$ transducers. All the patients were examined in the left lateral and supine positions with 2D, M-mode, pulsed, and color flow Doppler ECHO. Single lead electrocardiogram was recorded continuously. An average of at least 5 cardiac cycles was obtained for all measurements.

M-mode measurements and conventional Doppler ECHO examinations were performed based on the criteria of the American Society of Echocardiography and European Society of Echocardiography guidelines [18]. Left atrial (LA), LV end-systolic and end-diastolic dimensions were measured in the parasternal long-axis views. LVEF was estimated by Simpson's rule. Peak and mean transmitral pressures were measured via continuous wave Doppler. MVA was measured planimetrically in $2 \mathrm{D}$ views from the parasternal short axis and using the pressure half-time method by applying continuous wave Doppler during apical 4 -chamber view of the mitral valve. The area was calculated by the mean value of 2 measurements [19]. Pulmonary artery systolic pressure (PASP) was estimated by continuous-wave Doppler imaging using the Bernoulli equation [20].

\section{Tissue Doppler imaging}

Doppler tissue ECHO was performed using transducer frequencies between 3.5 to $4.0 \mathrm{MHz}$, by adjusting the spectral pulsed Doppler signal filters until a Nyquist limit of 15 to $20 \mathrm{~cm} / \mathrm{s}$ was reached, and using the minimal optimal gain. Five consecutive cycles were recorded with a frame rate greater than $150 \mathrm{fps}$. The monitor sweep speed was set at 50 to $100 \mathrm{~mm} / \mathrm{s}$ to optimize the spectral display of myocardial velocities. Every effort was made to align the pulsed wave cursor so that the Doppler angle of incidence was as close to 0 as possible to the direction of these walls. In the apical 4-chamber view, the pulsed Doppler sample volume was subsequently placed at the level of LV lateral and septal basal wall at end-expiration [21]. 


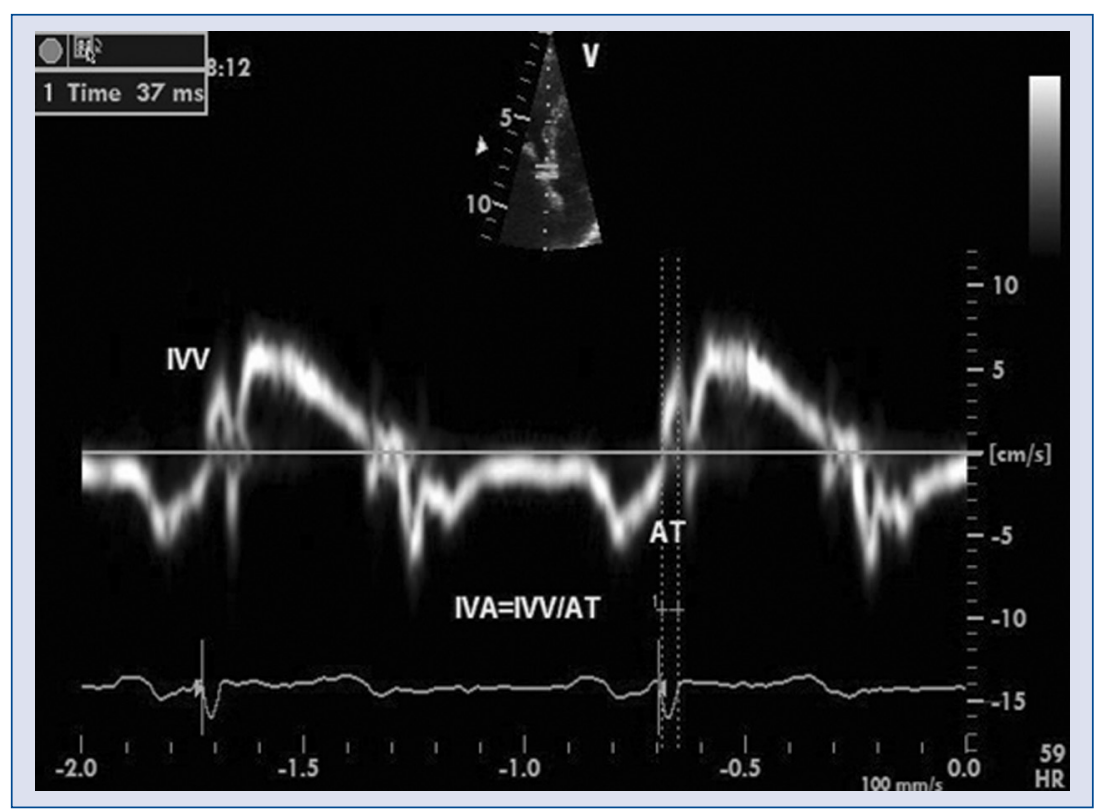

Figure 1. Tissue Doppler imaging-derived IVV and AT obtained from left ventricular septal basal wall of patients with mitral stenosis; IVV - peak myocardial velocity during isovolumic contraction; AT — isovolumic acceleration time; IVA - myocardial acceleration during isovolumic contraction.

Peak myocardial IVV, peak myocardial systolic velocity (Sm), peak early and late diastolic velocities ( $\mathrm{e}^{\prime}$ and a'), isovolumic AT, isovolumic contraction time (IVCT), isovolumic relaxation time (IVRT) and ejection time (ET) were measured. Myocardial performance index (MPI) was calculated as the sum of IVCT and IVRT divided by the ET. Myocardial acceleration during isovolumic contraction (IVA) was defined as the ratio of IVV divided by the AT (Fig. 1).

All the ECHO evaluations were performed by 2 different investigators. In order to detect the intraobserver variability, the first investigator repeated the ECHO measurements of 20 patients and the second investigator measured TDI-derived parameters of 20 patients to detect interobserver variability.

\section{Statistics analysis}

Statistical analyses were performed using the SPSS software version 17 . The variables were investigated using visual and analytical methods to determine whether they were normally distributed or not. Descriptive analyses were presented as mean \pm standard deviation (SD) and categorical variables were shown as percentages. Groups were compared using the Student's t-test, Mann-Whitney U test, one-way ANOVA, and Kruskal-Wallis test (MVA, mitral valve gradient, PASP, Sm, e', a', IVCT, IVRT, AT, IVV). Fisher's exact or $\chi^{2}$ tests was used to compare different groups. Mann-Whitney U test or Tukey's test was performed to determine the significance of pairwise differences using Bonferroni correction to adjust for multiple comparisons. Correlation analyses were derived by using Spearman test. An overall 5\% type-I error level was used to infer statistical significance.

\section{Results}

\section{Clinical properties}

Parameters such as age, gender, body mass index, heart rate, systolic blood pressure, diastolic blood pressure, smoking, hypertension, and diabetes mellitus were similar in both MS and healthy groups (Table 1). Of the MS patients, 52 were classified in New York Heart Association functional class I or II and 10 patients were in class III.

\section{Conventional echocardiographic parameters}

LVEF, LV end-diastolic and end-systolic diameters were similar in both the patient and the control groups. As an expected result, LA diameter and PASP were significantly higher in the patients with MS. LA diameter in patients with mild and moderate MS was significantly lower than those of the patients with severe MS $(p=0.004)$; however, there was no significant difference between the 
Table 1. Demographic characteristics and conventional echocardiographic parameters of the patients with mitral stenosis (MS) and healthy controls.

\begin{tabular}{|c|c|c|c|c|c|c|c|}
\hline & \multicolumn{7}{|c|}{ Severity of MS } \\
\hline & $\begin{array}{c}\text { Mild } \\
(n=21)\end{array}$ & $\begin{array}{l}\text { Moderate } \\
(n=21)\end{array}$ & $\begin{array}{l}\text { Severe } \\
(n=20)\end{array}$ & $\mathbf{P}$ & $\begin{array}{l}\text { MS total } \\
(n=62)\end{array}$ & $\begin{array}{l}\text { Control } \\
\text { groups } \\
(\mathrm{n}=32)\end{array}$ & $\mathbf{P}$ \\
\hline Age [years] & $40 \pm 9$ & $41 \pm 7$ & $36 \pm 7$ & 0.137 & $39 \pm 8$ & $36 \pm 8$ & 0.163 \\
\hline Gender (female) & $20(95.1 \%)$ & $19(90.59 \%)$ & $17(85 \%)$ & 0.541 & $56(90.3 \%)$ & $26(81.2 \%)$ & 0.177 \\
\hline Body mass index $\left[\mathrm{kg} / \mathrm{m}^{2}\right]$ & $29 \pm 6$ & $27 \pm 3$ & $27 \pm 4$ & 0.190 & $27 \pm 5$ & $28 \pm 4$ & 0.467 \\
\hline Systolic BP [mm Hg] & $122 \pm 12$ & $115 \pm 11$ & $115 \pm 9$ & 0.076 & $117 \pm 11$ & $118 \pm 10$ & 0.677 \\
\hline Diastolic BP [mm Hg] & $75 \pm 10$ & $71 \pm 9$ & $72 \pm 8$ & 0.360 & $72 \pm 9$ & $72 \pm 8$ & 0.812 \\
\hline Heart rate [beats/min] & $80 \pm 12$ & $78 \pm 10$ & $80 \pm 11$ & 0.606 & $80 \pm 12$ & $78 \pm 7$ & 0.378 \\
\hline Current smoking & $2(9.5 \%)$ & $1(4.8 \%)$ & $2(10 \%)$ & 0.790 & $5(8.1 \%)$ & $7(21.9 \%)$ & 0.060 \\
\hline Hypertension & $5(23.5 \%)$ & $5(23.8 \%)$ & $2(10 \%)$ & 0.437 & $12(19.4 \%)$ & $4(12.5 \%)$ & 0.402 \\
\hline Diabetes mellitus & $2(9.5 \%)$ & $1(4.8 \%)$ & 0 & 0.365 & $3(4.8 \%)$ & $0(0 \%)$ & 0.282 \\
\hline LVESD [mm] & $30 \pm 4$ & $27 \pm 4$ & $29 \pm 3$ & 0.121 & $29 \pm 4$ & $28 \pm 4$ & 0.272 \\
\hline LVEDD [mm] & $48 \pm 5$ & $47 \pm 5$ & $47 \pm 5$ & 0.738 & $47 \pm 5$ & $46 \pm 5$ & 0.184 \\
\hline LVEF [\%] & $63 \pm 3$ & $64 \pm 2$ & $64 \pm 3$ & 0.077 & $64 \pm 3$ & $65 \pm 3$ & 0.132 \\
\hline Left atrium diameter [mm] & $39 \pm 7$ & $41 \pm 4$ & $45 \pm 6^{*}$ & 0.003 & $42 \pm 7$ & $28 \pm 4$ & $<0.001$ \\
\hline $\begin{array}{l}\text { Pulmonary artery systolic } \\
\text { pressure }[\mathrm{mm} \mathrm{Hg}]\end{array}$ & $33 \pm 6$ & $39 \pm 10$ & $50 \pm 14$ & $<0.00^{* *}$ & $40 \pm 13$ & $26 \pm 4$ & $<0.001$ \\
\hline Mean mitral valve area $\left[\mathrm{cm}^{2}\right]$ & $1.8 \pm 0.1$ & $1.3 \pm 0.1$ & $0.8 \pm 0.1$ & $<0.001^{\#}$ & & & \\
\hline \multicolumn{8}{|l|}{$\begin{array}{l}\text { Transmitral diastolic gradient } \\
{[\mathrm{mm} \mathrm{Hg]}:}\end{array}$} \\
\hline Maximum & $11 \pm 4$ & $16 \pm 4$ & $27 \pm 8$ & $<0.001^{\#}$ & & & \\
\hline Mean & $6 \pm 2$ & $9 \pm 3$ & $18 \pm 6$ & $<0.001^{\#}$ & & & \\
\hline
\end{tabular}

${ }^{*} \mathrm{p}=0.004$ vs. mild and moderate; ${ }^{*} \mathrm{p}<0.01$ between all subgroups; ${ }^{*} \mathrm{p}<0.001$ between all subgroups; BP - blood pressure; LVEDD - left ventricular end-diastolic diameter; LVESD — left ventricular end-systolic diameter, LVEF — left ventricular ejection fraction

patients with mild and moderate MS. There was a stepwise increase in the PASP and diastolic transmitral gradients (maximum and mean) from mild to severe MS $(p<0.01$ and $p<0.001$, respectively). A stepwise decrease was found in the MVA from mild to severe MS $(\mathrm{p}<0.001)$ (Table 1$)$.

\section{TDI velocities}

All the TDI-derived global LV basal systolic (IVV, IVA, Sm) and diastolic velocities (e', a') were significantly decreased in the patients with MS compared to the healthy groups ( $p<0.001$, for all). In patients with MS, IVRT and MPI were significantly higher than the control group $(\mathrm{p}<0.001$, $\mathrm{p}=0.007$, respectively) and the ET was lower in the control group $(\mathrm{p}=0.018)$. The AT and IVCT were not different among the groups $(\mathrm{p}=0.432$, $\mathrm{p}=0.119$, respectively) (Table 2 ).

The IVA and Sm, which indicate LV systolic function, and MPI, which shows LV both systolic and diastolic function, did not differ among the 3 MS groups $(p=0.114, p=0.096$ and $p=0.238$, respectively). Excluding ET, time intervals such as
AT, IVRT and IVCT were not different among the MS groups $(p=0.005, p=0.922, p=0.192$ and $\mathrm{p}=0.498$, respectively). The IVV, e' and a' velocities in the patients with severe MS were significantly lower than those of the patients' with mild MS ( $<<0.01$, for all). However, there were no significant differences between the moderate and both mild and severe MS patients ( $p$ > 0.017). Agreement of intraobserver and interobserver variabilities were obtained for the IVA $(4 \pm 2 \%$ and $5 \pm 3 \%$, respectively).

\section{Correlation between TDI velocities and conventional echocardiographic parameters \\ MVA was positively correlated with both LV} diastolic velocities (e' and a') and IVV ( $p<0.01$, for all). However, there were no correlations between LV systolic velocities (IVA and Sm) and the MVA ( $>$ > 0.05). Mean transmitral gradient was weakly correlated with both IVV and e' $(\mathrm{p}<0.05)$. LA diameter and both systolic (IVV, IVA, Sm) and diastolic (e', a') velocities were shown to correlate $(\mathrm{p}<0.001$, for all). PASP was significantly associa- 
Table 2. Tissue Doppler-derived myocardial systolic and diastolic velocities obtained from the left ventricle.

\begin{tabular}{|c|c|c|c|c|c|c|c|}
\hline & \multicolumn{7}{|c|}{ Severity of mitral stenosis (MS) } \\
\hline & $\begin{array}{c}\text { Mild } \\
(n=21)\end{array}$ & $\begin{array}{l}\text { Moderate } \\
(\mathrm{n}=21)\end{array}$ & $\begin{array}{l}\text { Severe } \\
(n=20)\end{array}$ & $\mathbf{P}$ & $\begin{array}{l}\text { MS total } \\
(n=62)\end{array}$ & $\begin{array}{l}\text { Control } \\
\text { groups } \\
(\mathrm{n}=32)\end{array}$ & $\mathbf{P}$ \\
\hline IVV [cm/s] & $5.6 \pm 0.9$ & $5.2 \pm 0.8$ & $4.9 \pm 0.7^{*}$ & 0.018 & $5.2 \pm 0.8$ & $6.8 \pm 1.2$ & $<0.001$ \\
\hline Isovolumetric AT [ms] & $22 \pm 4$ & $22 \pm 4$ & $22 \pm 5$ & 0.922 & $22 \pm 4$ & $23 \pm 5$ & 0.432 \\
\hline IVA $\left[\mathrm{m} / \mathrm{s}^{2}\right]$ & $2.7 \pm 0.8$ & $2.5 \pm 0.6$ & $2.3 \pm 0.6$ & 0.114 & $2.5 \pm 0.7$ & $3.2 \pm 0.9$ & $<0.001$ \\
\hline $\mathrm{Sm}[\mathrm{cm} / \mathrm{s}]$ & $7.9 \pm 1.2$ & $7.3 \pm 1.3$ & $7.2 \pm 1.3$ & 0.096 & $7.5 \pm 1.3$ & $9.6 \pm 1.3$ & $<0.001$ \\
\hline $\mathrm{e}^{\prime}[\mathrm{cm} / \mathrm{s}]$ & $7.2 \pm 3.2$ & $5.4 \pm 1.4$ & $4.9 \pm 1.0^{*}$ & 0.013 & $5.8 \pm 2.3$ & $13.1 \pm 2.7$ & $<0.001$ \\
\hline $\mathrm{a}^{\prime}[\mathrm{cm} / \mathrm{s}]$ & $8.8 \pm 1.9$ & $7.8 \pm 1.7$ & $7.1 \pm 1.3^{*}$ & 0.008 & $7.9 \pm 1.8$ & $10.1 \pm 2.1$ & $<0.001$ \\
\hline Isovolumic contraction time [ms] & $64 \pm 16$ & $68 \pm 16$ & $63 \pm 15$ & 0.498 & $65 \pm 15$ & $70 \pm 14$ & 0.119 \\
\hline Isovolumic relaxation time [ms] & $81 \pm 22$ & $90 \pm 21$ & $81 \pm 26$ & 0.192 & $84 \pm 23$ & $68 \pm 13$ & $<0.001$ \\
\hline Ejection time [ms] & $294 \pm 27$ & $289 \pm 24^{\#}$ & $267 \pm 28^{*}$ & 0.005 & $284 \pm 29$ & $298 \pm 25$ & 0.018 \\
\hline Myocardial performance index & $0.50 \pm 0.1$ & $0.55 \pm 0.1$ & $0.55 \pm 0.1$ & 0.238 & $0.53 \pm 0.1$ & $0.47 \pm 0.1$ & 0.007 \\
\hline
\end{tabular}

${ }^{*} \mathrm{p}<0.01$ vs. mild; ${ }^{*} \mathrm{p}=0.010$ vs. severe; IVV — peak myocardial velocity during isovolumic contraction; AT - acceleration time; IVA - myocardial acceleration during isovolumic contraction; Sm — peak myocardial velocity during systole; $\mathrm{e}^{\prime}$ — peak myocardial velocity during early diastole; a' - peak myocardial velocity during atrial contraction

Table 3. Correlation between tissue Doppler imaging velocities and conventional echocardiographic parameters.

\begin{tabular}{|c|c|c|c|c|c|c|c|c|}
\hline & \multicolumn{2}{|c|}{ Mean MVA } & \multicolumn{2}{|c|}{ Mean gradient } & \multicolumn{2}{|c|}{ Left atrium diameter } & \multicolumn{2}{|c|}{ PASP } \\
\hline & $\mathbf{r}$ & p & $\mathbf{r}$ & p & $\mathbf{r}$ & p & $\mathbf{r}$ & p \\
\hline IVV & 0.387 & 0.002 & -0.260 & 0.042 & -0.522 & $<0.001$ & -0.565 & $<0.001$ \\
\hline IVA & 0.185 & 0.150 & -0.147 & 0.255 & -0.368 & $<0.001$ & -0.384 & $<0.001$ \\
\hline $\mathrm{Sm}$ & 0.220 & 0.085 & -0.190 & 0.138 & -0.541 & $<0.001$ & -0.537 & $<0.001$ \\
\hline $\mathrm{e}^{\prime}$ & 0.348 & 0.006 & -0.317 & 0.012 & -0.687 & $<0.001$ & -0.708 & $<0.001$ \\
\hline$a^{\prime}$ & 0.374 & 0.003 & -0.243 & 0.057 & -0.387 & $<0.001$ & -0.493 & $<0.001$ \\
\hline
\end{tabular}

MVA — mitral valve area; PASP — pulmonary artery systolic pressure; IVV — peak myocardial velocity during isovolumic contraction; IVA — myocardial acceleration during isovolumic contraction; $\mathrm{Sm}$ - peak myocardial velocity during systole; $\mathrm{e}^{\prime}-$ peak myocardial velocity during early diastole; $a^{\prime}$ - peak myocardial velocity during atrial contraction

ted with the parameters of LV systolic (IVV, IVA, Sm) and diastolic (e', a') function ( $p<0.001$, for all) (Table 3).

\section{Discussion}

In the light of these findings, it can be concluded that TDI-derived systolic and diastolic velocities are significantly reduced in the patients with MS and LV systolic dysfunction occurs independently from the severity of MS. To the best of our knowledge, this is the first study that evaluated the association between LV systolic velocities and severity of MS using TDI.

It is well known that MS affects the LV systolic function in various degrees. The probable causes can be listed as: chronically restricted
LV filling due to the structural abnormalities in the mitral valve, increased afterload, myocardial involvement during rheumatic fever, extension of the scar process from the mitral valve into the adjacent posterior basal myocardium, restriction or tethering of the posterobasal myocardium by the scarred mitral apparatus, abnormal interventricular septal motion associated with the right-ventricular overload, and the decrease in LV compliance $[2,4]$. Conventional ECHO methods such as M-mode and $2 \mathrm{D} \mathrm{ECHO}$ can detect one fourth of the LV dysfunction in MS [2-6]. In recent years, the studies using TDI [3-5] and strain/strain rate imaging $[6,7]$ demonstrated the presence of subclinical LV systolic dysfunction, which was not detected with the conventional ECHO methods in pure MS patients with preserved EF. Since the gold standard 
measurement of the ventricular contractility (end-systolic elastance) requires invasive quantification [22], and most of the contractile indexes (e.g., EF, MPI, and TDI-derived velocities or strain) are highly sensitive to loading conditions, it is thought that they might not reflect the intrinsic myocardial performance [17, 23, 24].

Isovolumic acceleration is a new tissue Doppler parameter for the assessment of systolic function of both left and the right ventricles [13, 14]. It remains unaffected by the changes in the preload and afterload within the physiological range [13-16] including age, sex and body mass index [25]. It can detect even small changes in the contractile function and is well correlated with the invasive or noninvasive measures of $\mathrm{LV} \mathrm{dP} / \mathrm{dt}[13,17]$. Therefore, IVA can be used as a valuable and easy parameter for the quantification of global systolic function in various cardiac diseases [26, 27].

The present study shows that isolated MS may lead to significant decrease of LV systolic and diastolic function. In the studies using TDI-derived parameters, systolic velocities were demonstrated to be significantly decreased in the healthy controls, as consistent with our study [7-9]. Similar to the study by Sengupta et al. [8], Ozdemir et al. [9] demonstrated that early and late diastolic myocardial velocities were decreased in MS. On the other hand, Dogan et al. [28] did not find any differences compared to the controls. Decreased LV pre-load, impaired compliance and increased LA afterload are known changes in MS. LA size increases with the LA volume and pressure, and is associated with the initial gain in the contractile shortening. However, with progressive dilatation of the LA, which eventually leads to a threshold fiber length, atrial shortening and contractility begin to decline. Beyond the threshold, further enlargement will only result in the deterioration of atrial function. This theory was supported by decreased e' and a' velocities, and the presence of positive correlation between this decrease and MVA in our study. Previous study demonstrated that MPI and IVRT were increased and ET was shortened [29] in the patients with MS compared to the controls. In consistent with the previous studies, the present study demonstrated that ET decreased as the severity of MS increased and, MPI and IVRT increased independently from the severity of MS.

Even though IVV, IVA and Sm velocities decrease in the patients with MS compared to the control group, this decrease is independent from the degree of MS. In addition, the fact that the systolic parameters such as IVA and Sm velocities are not correlated with MVA and the mean gradient, though weakly associated with IVV, indicates that the LV systolic dysfunction might be caused by an acute rheumatic fever leading to myocardial damage. The results of some studies $[12,30]$ were consistent with these findings in that the major determinant of the LV systolic dysfunction in isolated MS is impaired contractility and not the hemodynamic factors. Lee and Lee [12] evaluated the LV myocardium of 15 isolated MS patients using electron microscopy and demonstrated structural changes in LV independent from the $\mathrm{EF}$ and the degree of MS, determined by ECHO parameters. However, these patients with abnormal LV function always exhibit more extensive loss of myofibrils which results from either disproportion of the mitochondria-to-myofibril ratio or myofibrillar degeneration. In the study of Bilen et al. [30], which investigated the LV systolic function of mild, moderate and severe MS patients with preserved EF, using 2D speckle-tracking ECHO-derived strain; it was demonstrated that LV longitudinal function was decreased in patients with MS compared to the controls, however, this decrease was independent from the degree of MS. In our study, LV systolic function was evaluated globally with TDI-derived IVA and similar results were obtained. Furthermore, the patients were more evenly distributed into the groups in the present study.

Tayyareci et al. [26] investigated the IVA of right ventricle in prediction of the degree of MS and demonstrated that Sm, IVV and IVA of right ventricle significantly decreased in the patients with MS compared to the control group, however, they noted that only right ventricle and IVA can predict the degree of MS. Decreased IVA of LV was shown in MS patients in our study, although it was not associated with the degree of stenosis. This discrepancy might be caused by the classification differences; since Tayyareci et al. [26] grouped the MS patients into two groups (mild-moderate and severe) instead of three (mild, moderate and severe), which was helpful in the assessment of treatment strategies. Besides, the correlation of IVA with both LA diameter and PASP might be resulted from the increase in these two parameters due to hemodynamic effects of stenosis, instead of the systolic dysfunction of $\mathrm{LV}$.

\section{Limitations of the study}

The current study has some limitations. First, a small number of patients included in this study. Second, systolic function parameters were not compared with the parameters obtained from 
cardiac catheterization and magnetic resonance imaging. Third, the potential change in the IVA of LV was not assessed following the percutaneous balloon valvuloplasty.

\section{Conclusions}

In the light of these results, it is demonstrated that subclinical LV systolic dysfunction is present in all of the patients with MS and this condition is not affected by the degree of MS determined by ECHO.

\section{Conflict of interest: none declared}

\section{References}

1. Soler-Soler J, Galve E. Wordwide perspective of valve disease. Heart, 2000; 83: 721-725.

2. Colle JP, Rahal S, Ohayon J et al. Global left ventricular function and regional wall motion in pure mitral stenosis. Clin Cardiol, 1984; 7: 573.

3. Gaasch WH, Folland ED. Left ventricular function in rheumatic mitral stenosis. Eur Heart J, 1991; 12: 66-69.

4. Mohan JC, Khalilullah M, Arora R. Left ventricular intrinsic contractility in pure rheumatic mitral stenosis. Am J Cardiol, 1989; 64: 240-242.

5. Gash AK, Carabello BA, Cepin D, Spaan JF. Left ventricular ejection performance and systolic muscle function in patients with mitral stenosis. Circulation, 1983; 67: 148-154.

6. Heller SJ, Carleton RA. Abnormal left ventricular contraction in patients with mitral stenosis. Circulation, 1970; 42: 1099-1 110.

7. Ozer N, Can I, Atalar E et al. Leftventricular long-axis function is reduced in patients with rheumaticmitral stenosis. Echocardiography, 2004; 21: 107-112.

8. Sengupta PP, Mohan JC, Mehta V et al. Effects of percutaneous mitral commissurotomy on longitudinal left ventricular dynamics in mitral stenosis: Quantitative assessment by tissue velocity imaging. J Am Soc Echocardiogr, 2004; 17: 824-848.

9. Ozdemir K, Altunkeser BB, Gok H, Icli A, Temizhan A. Analysis of the myocardial velocities in patients with mitral stenosis. J Am Soc Echocardiogr, 2002; 15: 1472-1478.

10. Simsek Z, Karakelleoglu S, Gundugdu F et al. Evaluation of left ventriculat function with strain/strain rate imaging in patients with rheumatic mitral stenosis. Anadolu Kardiyol Derg, 2010; 10: 328-333.

11. Doğan S, Aydın M, Gursurer M, Dursun A, Onuk T, Madak H. Prediction of subclinical left ventricular dysfunction with strain rate imaging in patients with mild to moderate rheumatic mitral stenosis. J Am Soc Echocardiogr, 2006; 19: 243-248.

12. Lee YS, Lee CP. Ultrastructral pathological study of left ventricular myocardium in patients with isolated rheumatic mitral stenosis with normal or abnormal left ventricular function. Jpn Heart J, 1990; 31: 435-448.

13. Vogel M, Cheung M, Li J et al. Noninvasive assessment of left ventricular force-frequency relationships using tissue Doppler derived isovolumic acceleration: Validation in a animal model. Circulation, 2003; 107: 1647-1652.

14. Vogel M, Schmidt MR, Kristiansen SB et al. Validation of myocardial acceleration during isovolumic contraction as a novel noninvasive index of right ventricular contractility. Comparison with ventricular pressure-volume relations in an animal model. Circulation, 2002; 105: 1693-1699.
15. Dalsqaard M, Snyder EM, Kjaergaard J, Johnson BD, Hassager C, Oh JK. Isovolumic acceleration measured by tissue Doppler echocardiography is preload independent in healthy subjects. Echocardiography, 2007; 24: 572-579.

16. Duan YY, Harada K, Toyono M, Ishii H, Tamura M, Takada G. Effects on acute preload reduction on myocardial velocity during isovolumic contraction and myocardial acceleration in pediatric patients. Pediatr Cardiol, 2006; 27: 32-36.

17. Hashimoto I, Li X-K, Bhat AH, Jones M, Shan DJ. Quantitative assessment of regional peak myocardial acceleration during isovolumic contraction and relaxation times by tissue Doppler imaging. Heart, 2005; 91: 811-816.

18. Lang RM, Biering M, Devereux RB et al. Recommendations for chamber quantification: A report from the American society of echocardiography's guidelines and standards committee and the chamber quantification writing group, developed in conjunction with the European association of echocardiography, a branch of the European society of cardiology. J Am Soc Echocardiogr, 2005; 18: 1440-1463.

19. Baumgartner H, Hung J, Bermejo J et al. Echocardiographic assessment of valve stenosis: EAE/ASE recommendations for clinical practice. American Society of Echocardiography; European Association of Echocardiography. J Am Soc Echocardiogr, 2009; 22: 442.

20. Currie PJ, Seward JB, Chan KL et al. Continuous wave Doppler determination of right ventricular pressure: A simultaneous Doppler catheterization study in 127 patients. J Am Coll Cardiol, 1985; 6: 750-756.

21. Nagueh SF, Appleton CP, Gillebert TC et al. Recommendations for the evaluation of left ventricular diastolic function by echocardiography. Eur J Echocardiogr, 2009; 10: 165-193.

22. Katz A. Influence of altered inotropy and lusitropy in ventricular pressure-volume loops. J Am Coll Cardiol, 1988; 11: 438-445.

23. Abali G, Tokgozoglu L, Ozcebe OI, Aytemir K, Nazli N. Which Doppler parameters are load independent? A study in normal volunteers after blood donation. J Am Soc Echocardiogr, 2005; 18: 1260-1265.

24. Cheung MM, Smallhorn JF, Redington AN, Vogel M. The effects of preload changes in loading conditions and modulation of inotropic state on the myocardial performance index: Comparison with conductance catheter measurements. Eur Heart J, 2004; 25: 2238-2242.

25. Roche SL, Vogel M, Pitkanen O et al. Isovolumic acceleration at rest and during exercise in children. J Am Coll Cardiol, 2011; 57: 1100-1107.

26. Tayyareci Y, Nisanci Y, Umman B et al. Early detection of right ventricular systolic dysfunction by using myocardial acceleration during isovolumic contraction in patients with mitral stenosis. Eur J Echocardiogr, 2008; 9: 516-521.

27. Tayyareci Y, Yildirimturk O, Aytekin V, Demiroglu C, Aytekin S. Subclinical ventricular dysfunction in asymptomatic severe aortic regurgitaion patients with normal ejection fraction: A combined tissue Doppler and velocity vector imaging study. Echocardiography, 2010; 27: 260-268.

28. Dogan SM, Aydin M, Gursuer M, Dursun A, Cam F, Onuk T. Early detection of cardiac function by tissue Doppler imaging in patients with mitral stenosis and sinus rhythm. Arch Turk Soc Cardiol, 2006; 36: 358-362.

29. Ozdemir K, Altunkeser BB, Gok H, Icli A. Does the myocardial performance index affect pulmonary artery pressure in patients with mitral stenosis? A tissue Doppler imaging study. Echocardiography, 2003; 20: 249-256.

30. Bilen E, Kurt M, Tanboga IH et al. Severity of mitral stenosis and left ventricular mechanics: A speckle tracking study. Cardiology, 2011; 119: 108-115. 